\title{
ÍNDICE DE VERDOR DE LISIANTHUS (Eustoma grandiflorum (Raf.) Shinners) EN FUNCIÓN DE LA CONCENTRACIÓN DE 6-BENCILAMINOPURINA
}

\section{GREENNESS INDEX OF LISIANTHUS (Eustoma grandiflorum (Raf.) Shinners) AS A FUNCTION OF 6-BENZYLAMINOPURINE CONCENTRATION}

\author{
A. Belem G. Rojas-Morales ${ }^{1}$, Edgar J. Morales-Rosales ${ }^{2 *}$, E. Javier Morales-Morales ${ }^{2}$, \\ Gaspar Estrada-Campuzano², Omar Franco-Mora ${ }^{2}$ y José A. López-Sandoval ${ }^{2}$
}

\begin{abstract}
'Facultad de Ciencias Agrícolas, Universidad Autónoma del Estado de México (UAEMéx). El Cerrillo Piedras Blancas. 50200, Toluca, Estado de México. Tel. (722) 29655 18. 를 Estado de México, Tel. 2965531 Ext. 147 y 148.
\end{abstract}

*Autor para correspondencia (ejmoralesr@uaemex.mx)

\section{RESUMEN}

El manejo del color del follaje es de gran importancia en las especies ornamentales. El objetivo de la presente investigación fue evaluar en lisianthus (Eustoma grandiflorum (Raf.) Shinners) en precosecha el índice de verdor en los estratos inferior, medio y superior de la planta, número de botones por tallo floral, diámetro de tallo, altura de planta, área foliar y biomasa en función de aspersiones al follaje de $0.5,1.0$ y $1.5 \mathrm{mM}$ de 6 -bencilaminopurina (BAP) y de dos testigos, uno a base de aminoácidos y otro sin tratamiento; y en postcosecha el índice de verdor (unidades SPAD), color de pétalo y vida en florero en función de la dosis de 6-bencilaminopurina suministrada en el agua de riego y asperjándola al follaje. Para lo anterior, durante 2014 y 2015, en Toluca, México se realizaron dos experimentos. El diseño experimental en precosecha fue completamente al azar con cinco repeticiones y cinco tratamientos: $0.5,1$ y $1.5 \mathrm{mM}$ de BAP, un testigo con aminoácidos $(2 \mathrm{~mL}$ $L^{-1}$ ) y un testigo absoluto, mientras que en postcosecha se utilizó un arreglo factorial $2 \times 3$ en bloques completos al azar con tres repeticiones, siendo los factores de estudio la forma de aplicación (agua de riego y follaje) y dosis de BAP (1, 3 y $5 \mathrm{mM}$ ). Los datos se analizaron con análisis de varianza y las medias de los tratamientos se compararon con la prueba de Tukey $(0.05)$. En precosecha, mediante la aplicación de aminoácidos a razón $2 \mathrm{~mL} \mathrm{~L}^{-1}$ se alcanzó el máximo índice de verdor con 69.62 unidades SPAD, lo que superó en $16.65 \%$ al testigo. En postcosecha, la aplicación de BAP $1 \mathrm{mM}$ mantuvo el índice de verdor constante en cada uno de los muestreos realizados. El color del pétalo fue mayor con BAP $3 \mathrm{mM}$ asperjado al follaje $\left(b^{*}=23.37\right)$, valor que corresponde a morado intenso, y la vida en florero para este tratamiento fue de $15 \mathrm{~d}$, lo que superó significativamente al testigo.

Palabras clave: Eustoma grandiflorum, aminoácidos, citocininas, unidades SPAD, fitorreguladores, fotocolorímetro.

\section{SUMMARY}

The management of foliage color is of great importance in ornamental species. This study evaluated in pre-harvest lisianthus (Eustoma grandiflorum (Raf.) Shinners) the greenness index in the lower, middle and upper strata of the plant, number of buds per flowering stem, stem diameter, plant height, leaf area and biomass in response to leaf spraying of $0.5,1.0$ and 1.5 $\mathrm{mM}$ of 6 - benzylaminopurine (BAP). Data were compared to two controls, one based on amino acids and one with no treatment, and at post-harvest, the greenness index (SPAD units), petal color and vase life in response to 6-benzylaminopurine supplied in irrigation water and leaf spraying. During 2014 and 2015, in Toluca, Mexico, two experiments were carried out. The experimental design in pre-harvest was completely randomized with five replications and five treatments: $0.5,1$ and $1.5 \mathrm{mM}$ BAP, one control with amino acids $\left(2 \mathrm{~mL} \mathrm{~L}^{-1}\right)$ and one absolute control. For the post-harvest phase a factorial arrangement $(2 \times 3)$ under a randomized complete blocks design with three replications was used, where the study factors were the types of application (irrigation water and foliage) and BAP doses (1, 3 and $5 \mathrm{mM}$ ). Data were analyzed with analysis of variance and the means of treatments were compared with the Tukey (0.05) test. In pre-harvest, application of amino acids at $2 \mathrm{~mL} \mathrm{~L}^{-1}$ produced the maximum greenness index at $69.92 \mathrm{SPAD}$ units, exceeding the control by $16.65 \%$. In post-harvest, application of 1 mM BAP maintained the greenness index constant across samplings. Petal color was higher with $3 \mathrm{mM}$ BAP sprayed on the foliage $\left(b^{*}=23.37\right)$, which corresponds to intense purple, and the flower vase life for this treatment was $15 \mathrm{~d}$, significantly exceeding the control.

Index words: Eustoma grandiflorum, amino acids, cytokinins, SPAD units, plant growth regulators, photocolorimeter.

\section{INTRODUCCIÓN}

Lisianthus (Eustoma grandiflorum (Raf.) Shinners) es nativa del sur de Estados Unidos de América y norte de México. Es una planta herbácea de la familia de las Gencianáceas que forma roseta de hojas, sobre las cuales se desarrolla el tallo de consistencia rígida que alcanza alturas que oscilan entre 0.50 y $0.90 \mathrm{~m}$. Las flores de las plantas nativas presentan colores entre azul y morado, pero los híbridos tienen diversas tonalidades (Camargo et al., 2004).

En México, esta especie es de reciente utilización y su demanda en el mercado nacional va en aumento, por lo que se considera un cultivo con amplias perspectivas (Cruz-Crespo et al., 2006); sin embargo, se han visualizado algunos problemas en su producción. Esta especie ornamental presenta senescencia prematura de las hojas basales lo que provoca disminución significativa 
en el número de botones florales y en la calidad de la flor (Loyola y Vargas, 2005). El color amarillo de las hojas y de otros órganos (botones y tallos) es resultado de un proceso complejo que puede ser causado por diversos factores ambientales y hormonales (Reid, 2009). Los procesos de deterioro comienzan mucho antes de que se manifieste externamente esta fase. La senescencia ha sido estudiada siguiendo la disminución en la tasa fotosintética, degradación de pigmentos y cambios en los niveles de proteínas y ácidos nucleicos. Uno de los síntomas más visibles es el cambio de color como consecuencia de la degradación de las clorofilas (Thomas et al., 2003).

Existen numerosas referencias con relación al control de la senescencia por las citocininas (Haberer y Kieber, 2002). El uso de este regulador para retrasar la senescencia foliar tiene potencial para incrementar la productividad de cultivos, prolongar el almacenamiento postcosecha y aumentar la tolerancia al estrés (Lim et al., 2007). Las citocininas disminuyen durante la senescencia foliar, pero la aspersión de 6-bencilaminopurina (BAP) retrasa este proceso, lo cual puede tener aplicaciones en el control de la senescencia en pastos forrajeros, ornamentales de follaje y hortalizas verdes (González-Santos et al., 2009).

Los aminoácidos contribuyen a mejorar la calidad de flor; en este sentido, Triana y Gómez (2009) evaluaron la longitud y diámetro de tallo en rosa (Rosa sp.) y la longitud y diámetro de botón en clavel (Dianthus caryophyllus) y concluyeron que la adición de estos compuestos químicos a $0.50 \mathrm{~mL} \mathrm{~L}^{-1}$ de Starzyme (activador enzimático y hormonal) mejoró la calidad y presentación de ambas flores ornamentales.

Se reconoce desde hace tiempo que la medición de la clorofila foliar es una herramienta de suma utilidad en estudios de producción en plantas de ornato (Mirassón et al., 2010). El análisis de la intensidad del color verde en las hojas ha sido empleado en la evaluación del contenido de nitrógeno en la planta porque existe correlación directa entre la intensidad del color verde y el contenido de clorofila (Ribeiro et al., 2015). Los métodos tradicionales utilizados para determinar la cantidad de clorofila en la hoja requieren destrucción de muestras de tejido y mucho trabajo en los procesos de extracción y cuantificación (Argenta et al., 2001). Con los recientes avances de los medidores portátiles esta medición se convirtió en una tarea más rápida y de menor costo, lo que permite su uso como criterio de evaluación de la cantidad de nitrógeno en las plantas (Ribeiro et al., 2015).

El SPAD usa diodos emisores de 650 y $940 \mathrm{~nm}$ para emitir luz a través de la hoja. La luz en 650 nm está en la zona de las longitudes de onda asociadas con la absorción de la clorofila. Por su parte, la longitud de onda a 940 nm actúa como referencia interna. La diferencia de transmitancia a 650 y $940 \mathrm{~nm}$ produce el índice de verdor o unidades SPAD con el que luego se puede establecer una relación con el contenido de clorofila extraíble (Mirassón et al., 2010). Se trata de un medidor de mano, liviano, que no causa daño a las plantas y provee lecturas instantáneas, lo que evita los procedimientos de laboratorio que requieren tiempo y son costosos (Mirassón et al., 2010).

Los objetivos de la presente investigación fueron evaluar en precosecha el índice de verdor en los estratos inferior, medio y superior de la planta, número de botones por tallo floral, diámetro de tallo, altura de planta, área foliar y biomasa en función de aspersiones al follaje de 6-bencilaminopurina y aminoácidos; y evaluar en postcosecha el índice de verdor (unidades SPAD), color de pétalo y vida en florero en función de la aplicación de 6-bencilaminopurina suministrada en el agua de riego o asperjándola al follaje.

\section{MATERIALES Y MÉTODOS}

\section{Experimento 1}

Se realizó a partir del 20 de junio de 2014 en condiciones de cubierta plástica, en la Facultad de Ciencias Agrícolas de la Universidad Autónoma del Estado de México (19² $23^{\circ}$ $\mathrm{N}$; y $99^{\circ} 54^{\prime} \mathrm{O}$; a $2600 \mathrm{~m}$ de altitud), con temperatura media anual de $12.8^{\circ} \mathrm{C}$. Para formar las camas de siembra (10 $\mathrm{m}$ de largo $\times 1 \mathrm{~m}$ de ancho) se empleó agrolita, peat moss y tierra del lugar $(1: 1: 1 \mathrm{v} / \mathrm{v} / \mathrm{v})$, los cuales se desinfectaron con formaldehído mezclado con agua (1:10 v/v); el sustrato fue tapado con plástico y ventilado una semana antes de efectuar el trasplante.

Se utilizaron plántulas de lisianthus (E. grandiflorum) cv. $A B C$, resistente al 'roseteo' o 'arrosetamiento' (formación de conglomerados de hojas que impiden el desarrollo del tallo floral), con ciclo más corto que otros cultivares, de flor doble, número mayor de pétalos y tallos fuertes, de color azul-morado, que presenta vida en florero de entre 10 y 15 d. Las plántulas se adquirieron en Tenancingo, Edo. de México.

El 20 de junio de 2014 se realizó el trasplante cuando las plantas tenían cuatro hojas expandidas; esta actividad se efectuó por la mañana. El arreglo topológico fue $0.15 \mathrm{~m} \times$ $0.15 \mathrm{~m}$ entre plantas e hileras. Inmediatamente después se inició el riego por goteo. Al momento del trasplante se aplicaron $2 \mathrm{~mL} \mathrm{~L}^{-1}$ de sustancias fúlvicas (K-Tionic $($ ) para optimizar la asimilación de nutrimentos y $1 \mathrm{~mL} \mathrm{~L}^{-1}$ de micronutrientes (Razormin ${ }^{\circledR}$ ) para favorecer el enraizado y crecimiento tanto en longitud como en grosor de raíces. 
La nutrición de las plantas consistió en la aplicación de 12N-61P-00K (nitrógeno-fosforo-potasio, respectivamente) durante el primer mes a intervalos de $15 \mathrm{~d}$ en dosis de $300 \mathrm{mg} \mathrm{L}^{-1}$ de agua. A partir del segundo mes se utilizó la fórmula 19N-19P-19K a razón de $600 \mathrm{mg} \mathrm{L}^{-1}$ de agua y durante toda la estación de crecimiento se suministró nitrato de calcio $\left(800 \mathrm{mg} \mathrm{L}^{-1}\right)$, cada $10 \mathrm{~d}$.

Se realizaron aplicaciones preventivas para el control de hongos. Para Fusarium se alternó metalaxil (Ridomil gold $\AA$ ), Propamocarb clorhidrato (Previcur $\AA$ ) y Carbendazim (Derosal $\circledast$ ) cada $8 \mathrm{~d}$ en dosis de $1.5 \mathrm{~mL} \mathrm{~L}^{-1}$ de agua. Para Sclerotinia se utilizó Fosetil aluminio (Alietteß) cada $8 \mathrm{~d}$ en dosis de $1.5 \mathrm{~mL} \mathrm{~L}^{-1}$ de agua.

Se empleó la citocinina BAP con peso molecular de 225.25, en tres dosis 0.5, 1.0 y $1.5 \mathrm{mM}$ (BAP1, BAP2 Y BAP3, respectivamente) y un testigo absoluto y otro suministrando aminoácidos (ACTIVADORr, Bionet) (AA) a base de nitrógeno orgánico $6 \%$ (38\% de aminoácidos, $20 \%$ de carbono orgánico y $3 \%$ de ácidos húmicos) en dosis de 2 $\mathrm{mL} \mathrm{L} \mathrm{L}^{-1}$. La aplicación de los tratamientos se realizó a los 40 y 55 días después del trasplante. Los cinco tratamientos fueron evaluados en un diseño completamente aleatorizado con cinco repeticiones; la unidad experimental constó de 10 plantas.

Las variables en estudio fueron: 1) índice de verdor (IV), en unidades SPAD, se evaluó a los 70 días después del trasplante, y se determinó en hojas de los estratos bajo medio y alto de la planta: 1 a $25 \mathrm{~cm}, 26$ a $50 \mathrm{~cm}$ y 51 a $75 \mathrm{~cm}$, respectivamente, con un medidor SPAD 502 portátil, Konica Minolta, Japón; 2) altura de planta (AP), se midió con cinta métrica desde la base de la planta hasta el último botón floral y se expresó en centímetros; 3) diámetro de tallo (DT), se midió con vernier digital Mltutoyo 530-312, Japón, un centímetro arriba del cuello de la planta y se expresó en centímetros; 4) área foliar (AF), se midió con un integrador de área foliar modelo LI-COR $®-3100$ y se expresó en $\mathrm{cm}^{2}$; 5) número de botones por tallo floral (NBTF), se obtuvo contando el total de botones formados sin considerar tamaño; 6) biomasa (BIO), se obtuvo al secar los tallos florales en estufa de aire forzado Nahita 632 plus $65 \mathrm{~L}$, Japón, a $70^{\circ} \mathrm{C}$ hasta peso constante y se expresó en g. Cada variable se evaluó en cinco plantas de cada unidad experimental con sus respectivas repeticiones y se determinó el promedio.

Se efectuó el análisis de varianza y comparaciones de medias con la prueba de Tukey (0.05) mediante el paquete estadístico Statistical Analysis Systems (SAS Institute, 2004).

\section{Experimento 2}

Se llevó a cabo a partir del 3 de febrero de 2015 en el Laboratorio de Horticultura de la Universidad Autónoma del Estado de México. Se utilizaron tallos florales cortados de lisianthus Cv. ABC del tratamiento testigo, los cuales estaban sólo fertilizados. Para medir el índice de verdor se empleó un arreglo factorial $2 \times 3$, con dos factores de estudio (formas de aplicación y dosis de BAP), los niveles de forma de aplicación fueron: agua de riego y aspersión al follaje de BAP y los niveles de dosis de BAP fueron 1, 3 y $5 \mathrm{mM}$. El diseño experimental empleado fue bloques completos al azar con tres repeticiones. Cuando se incluyó el testigo se analizó con un diseño completamente aleatorizado. La unidad experimental fue una cubeta de $5 \mathrm{~L}$ con tres tallos de lisianthus. Cuando las pruebas de $\mathrm{F}$ fueron significativas se aplicó la prueba de Tukey, 0.05 (Fernández et al., 2010).

Las variables en estudio fueron: 1) índice de verdor (IV) en unidades SPAD, se determinó en hojas de los tres estratos de la planta: 1 a $25 \mathrm{~cm}, 26$ a $50 \mathrm{~cm}$ y 51 a $75 \mathrm{~cm}$ y se utilizó el promedio. Se realizaron muestreos cada tercer día (IV1, IV2...IV7), a partir del 5 de febrero de 2015 con medidor SPAD Konica Minolta 502 portátil; 2) el color del pétalo en cada tallo se determinó en la primera flor abierta con fotocolorímetro Chroma meter Konica Minolta CR-400, instrumento que proporcionó tres lecturas: $L^{*}$ indica la claridad del color, a* la proporción roja-verde y b* la proporción amarilla-azul. Los valores $L^{*}$ son positivos y se encuentran entre 0 para color negro ideal y 100 para el blanco ideal; el valor de $a^{*}$ cuando es positivo se acerca al rojo y cuando es negativo se acerca al verde; los valores $b^{*}$ cuando son positivos presentan color amarillo y cuando son negativos indican una tonalidad azul-morada; 3) vida en florero, consistió en contar el número de días a partir del corte hasta la senescencia en el proceso de maduración de las flores. La fecha final se consideró cuando las flores abiertas y los botones presentaron marchitez.

\section{RESULTADOS Y DISCUSIÓN}

\section{Experimento 1}

En el Cuadro 1 se observan diferencias significativas para todas las variables estudiadas, con excepción del índice de verdor en los estratos medio y superior. El coeficiente de variación (\%) osciló entre 5.7 para AP y 17.2 para NBTF.

\section{Índice de verdor y número de botones por tallo floral}

En el Cuadro 2 se aprecia que las plantas de lisianthus asperjadas con AA tuvieron en promedio los valores más 
elevados en unidades SPAD. El valor mostrado en esta variable superó en $7.8,8.3,8.4$ y $15.5 \%$ a los tratamientos BAP3, BAP1, BAP2 y testigo absoluto, respectivamente. Lo anterior sugiere la importancia de suministrar aminoácidos a esta especie, ya que el verdor del follaje les confiere a las plantas mayor atractivo ornamental (Ojeda et al., 2010). La mayor eficiencia de los AA exógenos pudo ser debida a la mayor absorción e incorporación por las plantas por la vía foliar e integrarse así al metabolismo vegetal (Arjona et al., 2004). Los AA no son nutrimentos; sin embargo, su aplicación foliar puede ser positiva para las plantas ya que participan en procesos metabólicos importantes, entre los que se encuentran la asimilación del amonio y procesos de transaminación (Taiz y Zeiger, 2002). Estudios realizados en Colombia indican que aplicaciones foliares de 2.5 $\mathrm{mL} \mathrm{L}^{-1}$ de un producto hecho a base de aminoácidos libres, efectuadas a los 45, 60 y 90 días después del trasplante, incrementaron el rendimiento de cebolla de bulbo (Allium cepa L.) en $11.2 \mathrm{t} \mathrm{ha}^{-1}(37 \%)$ con respecto a un testigo (Arjona et al., 2004).

El índice de verdor en las tres dosis de BAP fue igual estadísticamente en los tres estratos de la planta; sin embargo, este fitorregulador en las tres dosis superó al testigo absoluto (Cuadro 2). Por esta razón el BAP constituye una alternativa después de los aminoácidos para mantener el color verde del follaje. Resultados parcialmente similares a este estudio se obtuvieron en trigo (Triticum aestivum L.), ya que a los 41 días después de la siembra las hojas tratadas con BAP retrasaron su senescencia, conservaron $60 \%$ de la clorofila y $44 \%$ de las proteínas solubles to- tales en particular de la Rubisco; en contraste, las hojas del control perdieron $92 \%$ de su clorofila y más del $90 \%$ de proteínas en el mismo periodo (González-Santos et al., 2009). Viasus-Quintero et al. (2013), en un experimento donde evaluaron la aplicación de 6-bencilaminopurina en la producción de fresa (Fragaria) en dosis de 300, 600 y $900 \mathrm{mg} \mathrm{L}^{-1}$, encontraron que todas las dosis incrementaron el rendimiento, y este aumento estuvo ligado al tamaño de fruto. Por otro lado, el número de botones florales en BAP3, BAP2 y AA no fue diferente desde el punto de vista estadístico (Figura 1).

Al momento del corte el número de botones por tallo floral (NBTF) en el testigo fue menor en 44.83, 42.8, 38.46 y $30.4 \%$ con rerlación a BAP3, BAP2, AA y BAP1, respectivamente (Figura 1). Taiz y Zeiger (2002) indican que los AA, por ser los componentes básicos de las proteínas, intervienen en la formación de diversos tejidos y son importantes en procesos internos como la floración y fructificación. Por otro lado, plantas de chile Habanero (Capsicum chinense Habanero Group) mostraron un efecto diferencial positivo a la aplicación de reguladores de crecimiento, en especial a Maxigrow (producto que contiene BAP), el que promovió mayor desarrollo vegetativo y reproductivo, favoreciendo amarre de flores y frutos (Ramírez-Luna et al., 2005).

\section{Diámetro de tallo y altura de planta}

En la Figura 2 se observa que las plantas que se desarroIlaron con BAP3 tuvieron mayor altura de planta con relación a los tratamientos BAP2, BAP1, AA y testigo absoluto;

Cuadro 1. Fuentes de variación y nivel de significancia para diámetro de tallo (DT), altura de planta (AP), área foliar (AF), biomasa (BIO), número de botones por tallo floral (NBTF), índice de verdor (IV) en el estrato inferior (Inf), medio (Med), superior (Sup) y promedio (Prom) en lisianthus.

\begin{tabular}{|c|c|c|c|c|c|c|c|c|c|c|}
\hline FV & GL & DT & AP & $A F$ & $\mathrm{BIO}$ & NBTF & IV Inf & IV Med & IV Sup & IV Prom \\
\hline Tratamiento & 4 & $* \star *$ & $\star \star \star *$ & 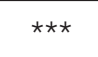 & $\star \star \star *$ & $* \star *$ & $\star \star \star *$ & $n s$ & ns & $\star \star \star \star$ \\
\hline CV (\%) & & 8.08 & 5.77 & 11.26 & 11.45 & 17.20 & 6.82 & 6.86 & 9.35 & 4.29 \\
\hline
\end{tabular}

***: significancia con $\mathrm{P} \leq 0.001$; ns: no significativo.

Cuadro 2. Índice de verdor (unidades SPAD) en los estratos inferior, medio, superior y promedio en hojas de lisianthus, en función de distintas dosis de la hormona 6-bencilaminopurina $(0.5,1.0$ y $1.5 \mathrm{mM})$ y aminoácidos (ACTIVADORr, Bionet).

\begin{tabular}{lcccc}
\hline \multicolumn{5}{c}{ Índice de verdor (Unidades SPAD) } \\
\hline Tratamiento & Estrato inferior & Estrato medio & Estrato superior & Promedio \\
\hline BAP3 & $65.96 \mathrm{ab}^{+}$ & $66.36 \mathrm{a}$ & $60.10 \mathrm{a}$ & $64.14 \mathrm{~b}$ \\
BAP2 & $64.82 \mathrm{ab}$ & $65.04 \mathrm{a}$ & $61.44 \mathrm{a}$ & $63.77 \mathrm{bc}$ \\
BAP1 & $61.86 \mathrm{~b}$ & $67.48 \mathrm{a}$ & $62.00 \mathrm{a}$ & $63.78 \mathrm{bc}$ \\
AA & $70.52 \mathrm{a}$ & $70.02 \mathrm{a}$ & $68.32 \mathrm{a}$ & $69.62 \mathrm{a}$ \\
Control & $52.08 \mathrm{c}$ & $66.20 \mathrm{a}$ & $58.06 \mathrm{a}$ & $58.78 \mathrm{c}$ \\
\hline
\end{tabular}

${ }^{\dagger}$ Promedios con letras iguales dentro de la misma columna no son estadísticamente diferentes de acuerdo con la prueba de Tukey 0.05. 
además, BAP3 y BAP2 presentaron promedios altos en diámetro de tallo con valores de 1.1 y $1.06 \mathrm{~cm}$, respectivamente. Lo anterior es relevante, ya que es una característica deseable para la comercialización de esta especie porque soporta el peso de las flores (De Pascale y Paradiso, 2007). Con respecto a la AP, ésta osciló entre 36.6 y 61.6 $\mathrm{cm}$ para el testigo absoluto y BAP3, respectivamente este rango que difiere de lo encontrado en un estudio de cuatro cultivares y cuatro distancias de plantación en lisianthus, ya que las AP estuvieron entre 45.5 y $72 \mathrm{~cm}$ en los cvs. Echo Misty Blue $(15 \times 15 \mathrm{~cm})$ y Balboa Purple $(12.5 \times 12.5$ $\mathrm{cm})$, respectivamente (Salazar, 2008).

\section{Área foliar y biomasa}

El área de la hoja en BAP3 fue superior estadísticamente a los demás tratamientos (Figura 3). La biomasa siguió una tendencia similar, ya que BAP en la dosis más elevada superó al testigo absoluto, AA y BAP1, y fue semejante estadísticamente a BAP2. En ambas variables todos los tratamientos superaron significativamente al testigo absoluto (Figura 3).

\section{Experimento 2}

En el Cuadro 3 se observa que no existieron diferencias significativas en ninguna variable para la forma de aplicación; sin embargo, hubo diferencias significativas entre dosis en todas las variables, con excepción de los muestreos IV2 e IV3. La interacción aplicación $\times$ dosis $(A \times D)$ no fue significativa en ninguna de las variables, con excepción de IV5 y IV6. El coeficiente de variación (\%) fue 2.9 para IV2 y 3.48 para IV7.

\section{Índice de Verdor}

No existió diferencia en el índice de verdor al aplicar 6-bencilaminopurina en agua o asperjada al follaje (Cuadro 3). Con relación a las dosis de BAP, con 1 mM en promedio se lograron los valores altos de unidades SPAD en cada uno de los muestreos realizados. En este sentido, 11 días después de la aplicación de esta citocinina, la pérdida de verdor fue de 8.7, 14.5 y $15.4 \%$, para las dosis de BAP $1 \mathrm{mM}, 3 \mathrm{mM}$ y $5 \mathrm{mM}$, respectivamente, lo cual representa menos degradación de clorofila con la dosis de $1 \mathrm{mM}$ de BAP (Liu et al., 2012).

En la Figura 4(A) se presenta la interacción aplicación $\times$ dosis para el muestreo del 13 de febrero de 2013 (IV5). Se aprecia que con los tratamientos BAP $1 \mathrm{mM}$ disuelto en agua y BAP $3 \mathrm{mM}$ asperjado al follaje se encontraron los máximos valores para el índice de verdor con 64.8 y 64.2, respectivamente, mientras que en la Figura 4(B) con $1 \mathrm{mM}$ de BAP disuelto en agua el índice de verdor fue de 62.6 y de 60.9 cuando se asperjó BAP al follaje de la planta. No obstante, en ambas formas de aplicación el índice de verdor no fue consistente y varió con las dosis (Figura 5A y 5B). En este sentido, Morales-Pérez et al. (2014) en gerbera (Gerbera jamesonii) cv. Dream no encontraron una tendencia lineal al aplicar ácido giberélico y salicílico aplicados al sustrato y follaje.

Las lecturas SPAD de ambas combinaciones fueron más altas comparadas con otros índices de verdor estimados en diversas ornamentales; por ejemplo en Lilium híbrido oriental en postcosecha se encontraron en promedio 51.6

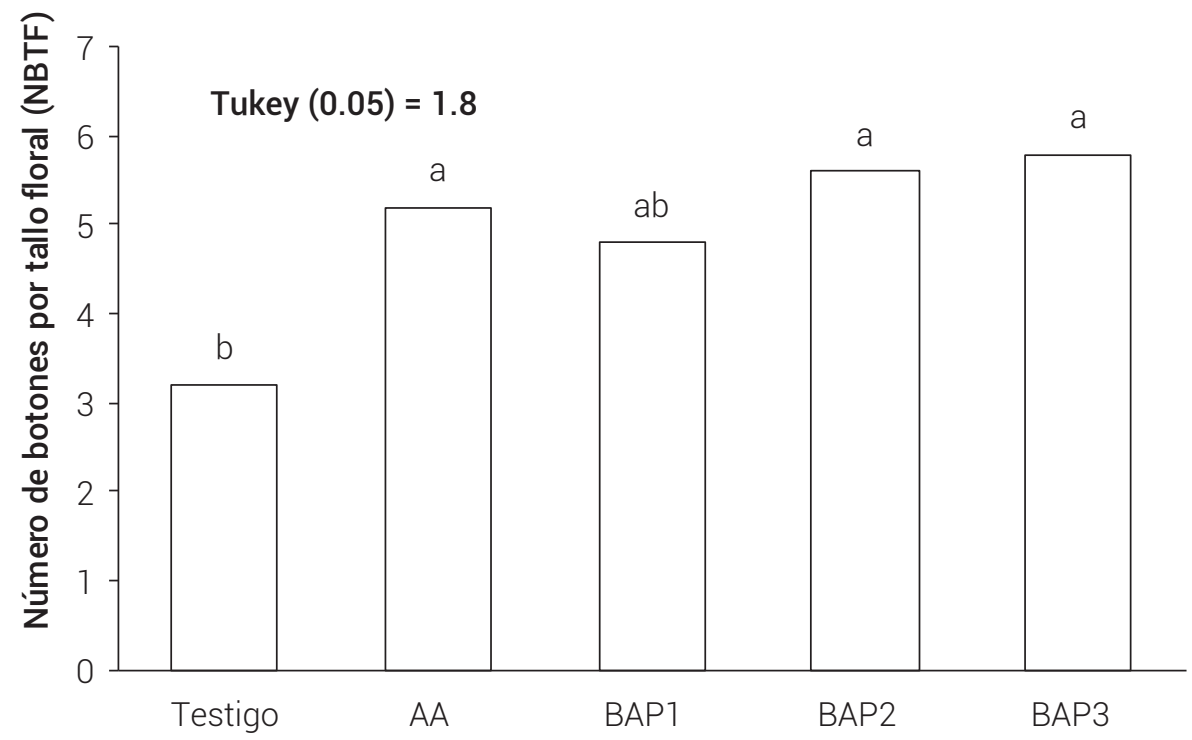

Figura 1. Número de botones por tallo floral de lisianthus, en función de diferentes dosis de 6-bencilaminopurina y aminoácidos (ACTIVADORr, Bionet). Letras iguales en las barras no son significativas de acuerdo con la prueba de Tukey (0.05). 


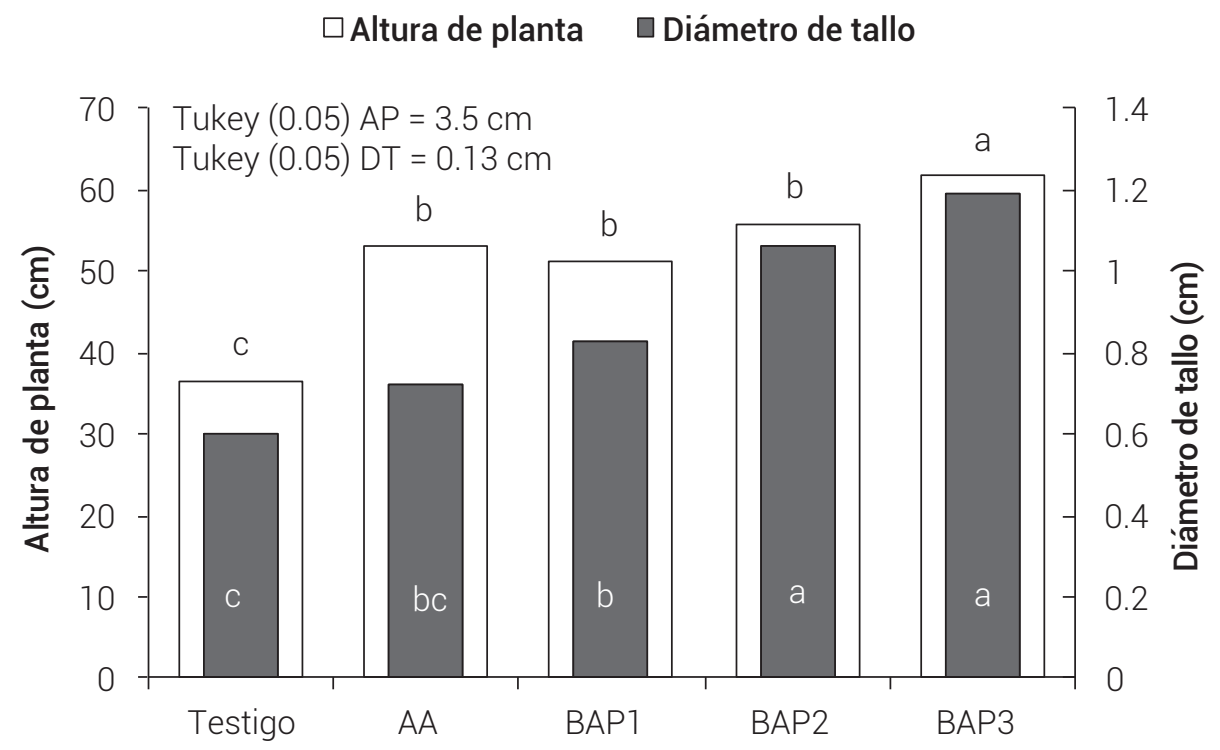

Figura 2. Altura de planta (AP) y diámetro de tallo (DT) de lisianthus en función de distintas dosis de 6-bencilaminopurina y aminoácidos (ACTIVADORr, Bionet). Letras iguales dentro de cada tipo de barra no son significativamente diferentes de acuerdo con la prueba de Tukey (0.05).

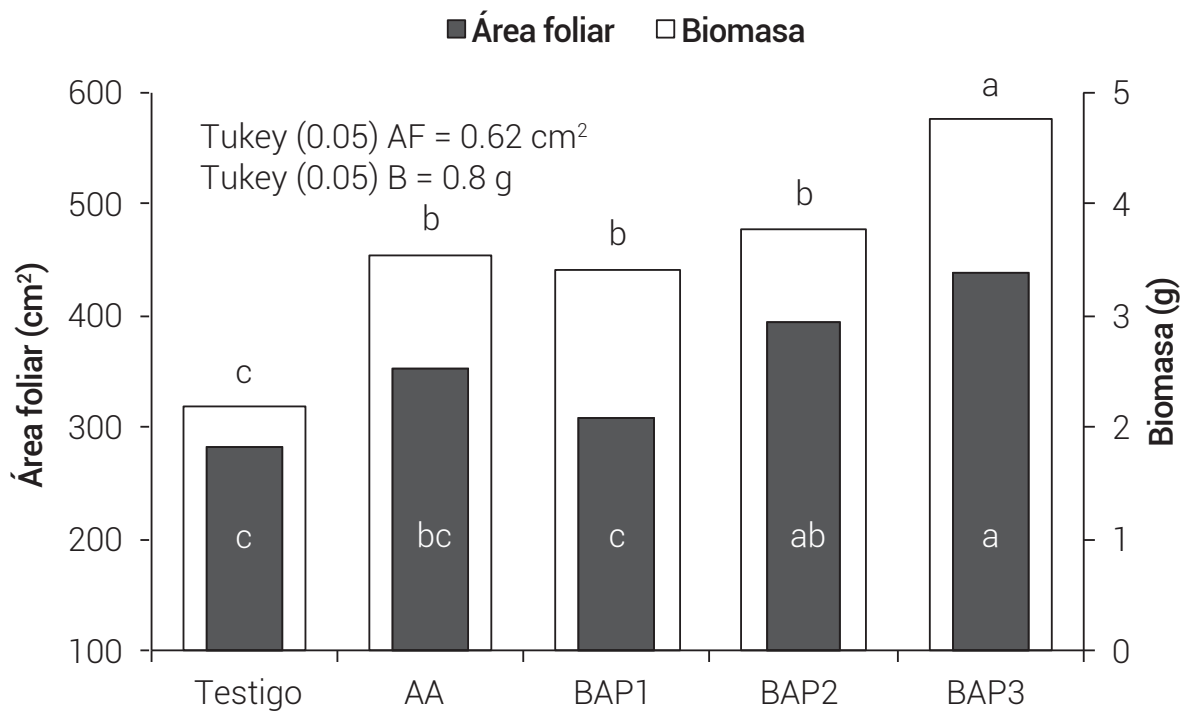

Figura 3. Área foliar (AF) y biomasa (B) de lisianthus en función de diferentes dosis de 6-bencilaminopurina y aminoácidos (ACTIVADORr, Bionet). Letras iguales dentro de cada tipo de misma barra no son significativamente diferentes de acuerdo con la prueba de Tukey (0.05).

unidades SPAD en los cultivares Star fighter y Star gazer (Mandujano-Piña et al., 2012).

\section{Vida en florero}

Se registró variación significativa entre los tratamientos evaluados en la vida en florero (Cuadro 4). El máximo valor fue encontrado en BAP aplicado en el agua de riego $1 \mathrm{mM} \mathrm{y}$ en BAP asperjado al follaje en dosis de $3 \mathrm{mM}$ (15 d), mientras que el valor mínimo fue obtenido por el testigo (11 d).
La vida en florero en el cv. ABC osciló entre 11 y 15 d, la cual supera parcialmente a otras especies florícolas como rosa y gladiola (Gladiolus) (7 a 15 d) y es buen indicador para recomendar su producción como flor de corte (Jamal Uddin et al., 2013).

\section{Color de pétalo}

En el Cuadro 4 se aprecia que no hubo significancia estadística para la variable $L^{*}$. En las características $a^{*}$ y b* 
Cuadro 3. Significancia estadística y separación de medias de dos formas de aplicación (al agua de riego y asperjado al follaje) y tres dosis de 6 bencilaminopurina en lisianthus $c v$. ABC en relación al índice de verdor.

\begin{tabular}{|c|c|c|c|c|c|c|c|}
\hline \multirow{2}{*}{ Factor } & \multicolumn{7}{|c|}{ Fecha de muestreo } \\
\hline & IV1 05-feb & IV2 07-feb & IV3 09-feb & IV4 11-feb & IV5 13-feb & IV6 15-feb & IV7 17-feb \\
\hline Aplicación (A) & ns & ns & ns & ns & ns & ns & ns \\
\hline Agua & $68.32 \mathrm{a}$ & 65.59 a & $64.19 a$ & 63.28 a & $61.41 \mathrm{a}$ & 59.23 a & $28.21 \mathrm{a}$ \\
\hline Aspersión & $66.84 a$ & 65.31 a & $64.03 \mathrm{a}$ & $62.15 \mathrm{a}$ & $60.92 \mathrm{a}$ & $58.53 \mathrm{a}$ & $28.54 \mathrm{a}$ \\
\hline Tukey (0.05) & 2.32 & 2.04 & 2.20 & 2.26 & 2.08 & 2.13 & 1.03 \\
\hline Dosis (D) & $\star$ & ns & ns & * & $* *$ & $\star *$ & $* *$ \\
\hline BAP $1 \mathrm{mM}$ & $67.77 \mathrm{ab}$ & $66.94 \mathrm{a}$ & 65.43 a & $63.92 \mathrm{a}$ & 63.27 a & $61.82 \mathrm{a}$ & $30.75 a$ \\
\hline BAP $3 \mathrm{mM}$ & 69.34 a & 65.98 a & 64.69 a & $63.77 \mathrm{ab}$ & $61.44 \mathrm{ab}$ & $59.27 \mathrm{a}$ & $29.49 a$ \\
\hline BAP $5 \mathrm{mM}$ & $65.63 c$ & $63.96 \mathrm{a}$ & 62.21 a & 60.46 b & $58.79 b$ & $55.54 b$ & $24.89 b$ \\
\hline Tukey (0.05) & 3.50 & 3.07 & 3.32 & 3.88 & 3.14 & 3.21 & 1.56 \\
\hline$A \times D$ & ns & ns & ns & ns & $\star *$ & * & ns \\
\hline CV (\%) & 3.28 & 2.97 & 3.27 & 3.44 & 3.25 & 3.45 & 3.48 \\
\hline
\end{tabular}

*: $\mathrm{P} \leq 0.05 ; * *: \mathrm{P} \leq 0.01 ; \mathrm{ns}$ : no significativo. Letras iguales para cada factor, en cada columna, no son significativamente diferentes de acuerdo con la prueba de Tukey (0.05).
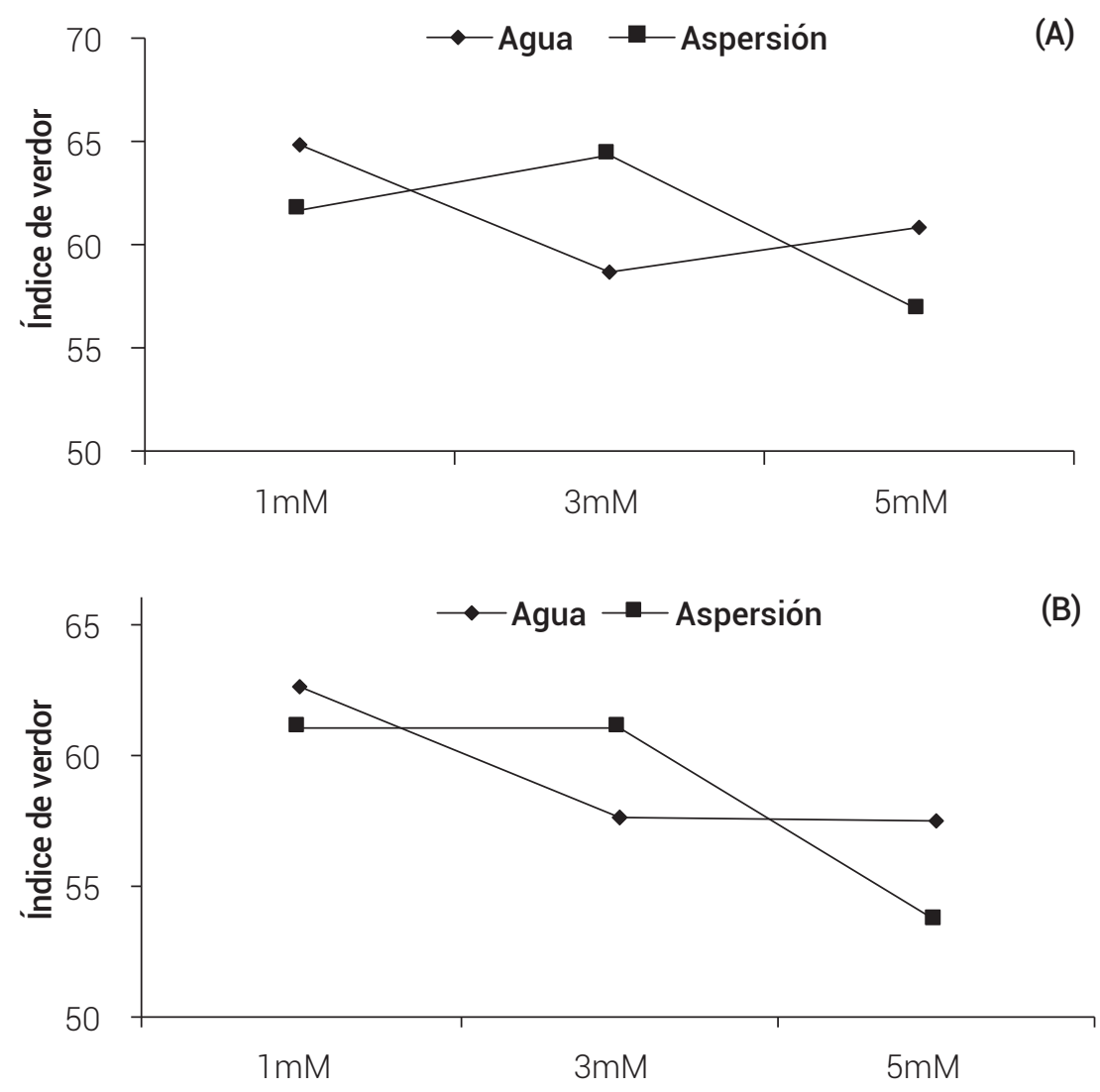

Figura 4. Interacción formas de aplicación × dosis de 6-bencilaminopurina, en los muestreos IV5 (A) e IV 6 (B). 
Cuadro 4. Características colorimétricas y vida en florero de lisianthus en función de diferentes dosis de la hormona 6-bencilaminopurina (BAP).

\begin{tabular}{lcccc}
\hline Tratamiento & $\mathrm{L}^{*}$ & $\mathrm{a}^{*}$ & $\mathrm{~b}^{*}$ & Vida en florero (días) \\
\hline Testigo & $46.13 \mathrm{a}$ & $14.63 \mathrm{c}$ & $-13.31 \mathrm{~b}$ & $11 \mathrm{c}$ \\
BAP Agua 1 mM & $45.66 \mathrm{a}$ & $25.35 \mathrm{a}$ & $-22.54 \mathrm{a}$ & $15 \mathrm{a}$ \\
BAP Agua 3 mM & $54.05 \mathrm{a}$ & $15.95 \mathrm{bc}$ & $-12.0 \mathrm{~b}$ & $13 \mathrm{~b}$ \\
BAP Agua 5 mM & $54.43 \mathrm{a}$ & $16.45 \mathrm{bc}$ & $-13.27 \mathrm{~b}$ & $13 \mathrm{~b}$ \\
BAP Asp. $1 \mathrm{mM}$ & $45.81 \mathrm{a}$ & $21.65 \mathrm{ab}$ & $-19.01 \mathrm{ab}$ & $13 \mathrm{~b}$ \\
BAP Asp. 3 mM & $45.78 \mathrm{a}$ & $25.78 \mathrm{a}$ & $-23.37 \mathrm{a}$ & $15 \mathrm{a}$ \\
BAP Asp. 5 mM & $44.63 \mathrm{a}$ & $18.32 \mathrm{bc}$ & $-14.51 \mathrm{~b}$ & $13 \mathrm{~b}$ \\
Significancia & $\mathrm{ns}$ & $* *$ & $* *$ & $* *$ \\
Tukey, 0.05 & 12.56 & 6.21 & 7.25 & 1.0 \\
CV $(\%)$ & 11.18 & 13.47 & 13.39 & 2.0 \\
\hline
\end{tabular}

$\mathrm{L}^{*}=$ luminosidad de color (0: negro, 100: blanca); $\mathrm{a}^{*}$ : valores positivos indican rojo, valores negativos indican verde; $b^{*}$ : valores positivos indican amarillo, valores negativos indican azul.

existieron diferencias significativas. El mayor valor de $a^{*}$ fue obtenido con BAP suministrado en agua 1 mM (25.3) y el más bajo se presentó en el testigo (14.6). El valor b* fue máximo (-23.3) en el tratamiento BAP asperjado al follaje (3 mM), mientras que los valores menos negativos -12.0 -13.3 y -13.2 fueron para BAP en agua $3 \mathrm{mM}$, testigo y BAP en agua $5 \mathrm{mM}$, respectivamente. En este orden de ideas, cuando los valores de b* son más negativos el color azulmorado que presentan los pétalos es más intenso, por lo que BAP asperjado al follaje $3 \mathrm{mM}$ de BAP disuelto en agua en $1 \mathrm{mM}$ le confirió a los pétalos color más intenso (Jamal Uddin et al., 2013), característica importante en la comercialización de esta especie ornamental.

\section{CONCLUSIONES}

En precosecha, la aplicación de $2 \mathrm{~mL} \mathrm{~L}^{-1}$ de aminoácidos lograron el máximo valor de unidades SPAD por lo que es la mejor opción para mantener verde el follaje de lisianthus. En postcosecha no existió efecto en las formas de aplicación de BAP a las plantas; sin embargo, todas las dosis ensayadas incrementaron la vida en florero de esta especie. La adición de BAP 1 mM en agua de riego y BAP $3 \mathrm{mM}$ asperjada al follaje afectaron positivamente el color de los pétalos, mostrando color azul-morado más intenso, condición que le confiere mayor oportunidad de venta en la comercialización.

\section{BIBLIOGRAFÍA}

Argenta G., P. R. Ferreira da Silva, C. G. Bortolini, E. L. Forsthofer e M. L. Strieder (2001) Relação da leitura do clorofilômetro com os teores de clorofila extraível e de nitrogênio na folha de milho. Revista Brasileira de Fisiologia Vegetal 13:158-167.

Arjona D. H., J. E. Herrera B., J. A. Gómez G., y J. Ospina A. (2004) Evaluación de la aplicación de urea, melaza y aminoácidos sobre el crecimiento y rendimiento de la cebolla de bulbo (Allium cepa L. Grupo cepa) híbrido yellow granex, en condiciones de la Sabana de Bogotá. Agronomía Colombiana 22:177-184.

Camargo M. S., L. K. Shimizu, M. A. Saito, C. H. Kameoka, S. C. Mello e Q. A. C. Carmello (2004) Crescimento e absorção de nutrientes pelo Lisianthus (Eustoma grandiflorum) cultivado em solo. Horticultura Brasileira 22:143-146. http://dx.doi.org/10.1590/S010205362004000100030.

Cruz-Crespo E., L. Arévalo-Galarza, R. Cano-Medrano y E. A. Gaytán-Acuña (2006) Soluciones pulso en la calidad postcosecha de lisianthus (Eustoma grandiflorum Raf.) cv. 'Echo Blue'. Agricultura Técnica en México 32:191-200.

De Pascale S. y R. Paradiso (2007) Programación de la producción de Lisianthus russellianus L. Horticultura Internacional 59:50-53.

Fernández E. R., A. Trapero y J. Domínguez (2010) Experimentación en Agricultura. Junta de Andalucía. Consejería de Agricultura y Pesca. Sevilla, España. 350 p.

González-Santos R., A. Delgado-Alvarado, H. A. Zavaleta-Mancera y B. E. Herrera-Cabrera (2009) La citocinina BAP promueve la acumulación de hexosas e incrementa la actividad de fosfoenolpiruvato carboxilasa y fosfoenolpiruvato carboxicinasa durante el retraso de la senescencia foliar de trigo. Agrociencia 43:379-391.

Haberer G. and J. J. Kieber (2002) Cytokinins. New insights into a classic phytohormone. Plant Physiology 128:354-362. https://doi. org/10.1104/pp.010773.

Jamal Uddin A. F. M., M. S. Islam, H. Mehraj, M. Z. K. Roni and S. Shahrin (2013) An evaluation of some Japanese lisianthus (Eustoma grandiflorum) varieties grown in Bangladesh. The Agriculturists 11:56-60. http://dx.doi.org/10.3329/agric.v17i1.15243.

Lim P. 0., H. J. Kim and H. G. Nam (2007) Leaf senescence. Annual Review of Plant Biology 58:115-136. doi: 10.1146/annurev.arplant.57.032905.105316.

Liu Z. A., J. P. Yang and Z. C. Yang (2012) Using a chlorophyll meter to estimate tea leaf chlorophyll and nitrogen contents. Journal of Soil Science and Plant Nutrition 12:339-348. http://dx.doi. org/10.4067/S0718-95162012000200013.

Loyola N. y J. Vargas (2005) Comparación de los efectos de los preservantes en postcosecha de lisianthus (Eustoma grandiflorum L..). Agro Sur 33:9-19.

Mandujano-Piña M., M. T. Colinas-León, A. M. Castillo-González, I. AlíaTejacal y L. A. Valdez-Aguilar (2012) Cobalto como retardante de la senescencia de Lilium híbrido oriental en postcosecha. Revista Chapingo Serie Horticultura 18:239-252. http://dx.doi. org/10.5154/r.rchsh.2010.09.034.

Mirassón H. R., M. L. Faraldo, M. N. Fioretti, M. Miravalles y R. E. Brevedan 
(2010) Relaciones entre el índice de verdor y el nivel hídrico foliar en trigo con diferentes sistemas de labranza. Phyton 79:183-187.

Morales-Pérez E., E. J. Morales-Rosales, O. Franco-Mora, D. J. Pérez-López, A. González-Huerta y E. Urbina S. (2014) Producción de flores de Gerbera jamesonii cv. 'Dream' en función de los ácidos giberélico y salicílico. Phyton 83:333-340.

Ojeda M., N. Mogollón, M. Pérez C. y N. Maciel (2010) Efecto de la irradiancia sobre el crecimiento y la clorofila de Billbergia pyramidalis (Sims) Lindley. Boletín del Centro de Investigaciones Biológicas $44: 7-26$

Ramírez-Luna E., C. C. Castillo-Aguilar, E. Aceves-Navarro y E. Carrillo-Avila (2005) Efecto de productos con reguladores de crecimiento sobre la floración y amarre de fruto en chile 'Habanero'. Revista Chapingo Serie Horticultura 11:93-98.

Reid M. S. M. (2009) Poscosecha de las Flores Cortadas. Manejo y Recomendaciones. Traducción al español por Marta Pizano, Ediciones Hortitecnia Ltda. Bogotá, Colombia. 38 p.

Ribeiro C. A., I. Katz, A. P. Souza y R. A. Martínez U. (2015) Índice SPAD en el crecimiento y desarrollo de plantas de lisianthus en función de diferentes dosis de nitrógeno en ambiente protegido. IDESIA (Chile) 33:97-105. http://dx.doi.org/10.4067/S071834292015000200012
Salazar C. H. A. (2008) Evaluación de Materiales Genéticos del Cultivo de Lisianthus (Eustoma grandiflorum), bajo Diferentes Densidades de siembra, en Condiciones Ambientales Controladas en la Región de El Tejar, Chimaltenango. Fondo Nacional de Ciencia y Tecnología (FONACYT)-Secretaria Nacional de Ciencia y Tecnología (SENACYT)-Consejo Nacional de Ciencia y Tecnología (CONCYT). Guatemala. $112 \mathrm{p}$.

SAS Institute (2004) SAS/STAT User's Guide, Version 8.02. SAS Institute, Inc. Cary, NC, USA

Taiz L. and E. Zeiger (2002) Plant Physiology. 3rd edition. Sinauer Associates Inc. Sunderland, MA, USA. $623 \mathrm{p}$.

Triana C. F. y C. Gómez (2009) Evaluación del activador enzimático y hormonal STARZYME en los cultivos de rosa (Rosa sp.) y clavel (Dianthus caryophyllus), bajo invernadero en la sabana de Bogotá. Inventum 6:58-65.

Thomas H., H. J. Ougham, C. Wagstaff and A. D. Stead (2003) Defining senescence and death. Journal of Experimental Botany 54:11271132.

Viasus-Quintero G., J. Álvarez-Herrera y O. Alvarado-Sanabria (2013) Efecto de la aplicación de giberelinas y 6-bencilaminopurina en la producción y calidad de fresa (Fragaria $x$ ananassa Duch). Bioagro 25:195-200. 
